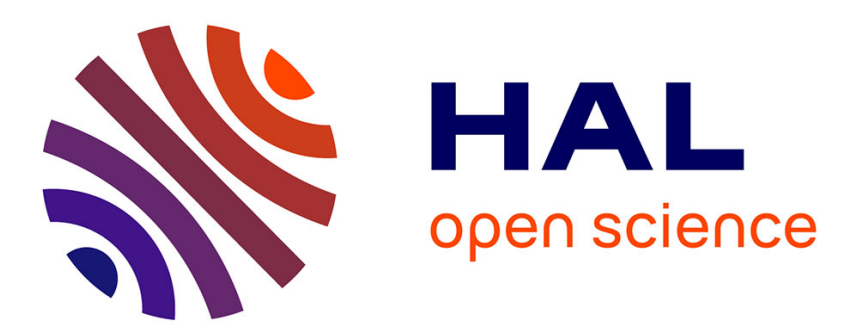

\title{
Emergence of a periodic mode in the so-called turbulent region in a circular Couette flow
}

\author{
Bernard Perrin
}

\section{To cite this version:}

Bernard Perrin. Emergence of a periodic mode in the so-called turbulent region in a circular Couette flow. Journal de Physique Lettres, 1982, 43 (1), pp.5-10. 10.1051/jphyslet:019820043010500 . jpa00232002

\section{HAL Id: jpa-00232002 https://hal.science/jpa-00232002}

Submitted on 1 Jan 1982

HAL is a multi-disciplinary open access archive for the deposit and dissemination of scientific research documents, whether they are published or not. The documents may come from teaching and research institutions in France or abroad, or from public or private research centers.
L'archive ouverte pluridisciplinaire HAL, est destinée au dépôt et à la diffusion de documents scientifiques de niveau recherche, publiés ou non, émanant des établissements d'enseignement et de recherche français ou étrangers, des laboratoires publics ou privés. 
Classification

Physics Abstracts

$47.20-47.25 \mathrm{~F}$

\title{
Emergence of a periodic mode in the so-called turbulent region in a circular Couette flow
}

\author{
B. Perrin \\ Groupe de Physique des Solides de l'Ecole Normale Supérieure $\left({ }^{*}\right)$, \\ 24, rue Lhomond, 75231 Paris Cedex 05, France
}

(Reçu le 25 juillet 1981, révisé le 4 novembre, accepté le 6 novembre 1981)

\begin{abstract}
Résumé. - Nous avons étudié la transition vers la turbulence dans l'hélium liquide normal en écoulement de Couette cylindrique jusqu'à des nombres de Reynolds $R=500 R_{\mathrm{c}}\left(R_{\mathrm{c}}\right.$ étant le nombre critique d'apparition de l'instabilité de Taylor) et pour de faibles facteurs de forme $\Gamma(\Gamma=l / d$ où $l$ est la hauteur de fluide et $d$ l'intervalle entre les cylindres). Pour $d$ suffisamment élevé et pour des nombres de Reynolds où l'on considère l'écoulement turbulent, un mode périodique apparaît et demeure jusqu'aux valeurs maxima de $R$. Nous avons étudié la dépendance de ce mode en $d$ et en température $T$. Nous suggérons que l'existence de ce mode est liée à la formation d'une nouvelle structure à couches limites.
\end{abstract}

\begin{abstract}
The transition to turbulence is studied in normal fluid He contained between concentric cylinders with the inner cylinder rotating, for high Reynolds numbers $R$ up to $500 R_{\mathrm{c}}\left(R_{\mathrm{c}}\right.$ is the critical Reynolds number for the Taylor instability) and for small aspect ratios $\Gamma(\Gamma=l / d=$ fluid height/gap between cylinders). For sufficiently high values of $d$ and in a range of $R$ where the flow is considered as turbulent, a periodic mode appears and remains up to the maximum value of $R$. The $T$ - and $d$-dependence of the mode has been studied. We suggest that this mode is related to a change in a boundary layers structure.
\end{abstract}

Circular Couette flows are presently, with Rayleigh-Bénard experiments, among the important experiments to study the successive instabilities, which appear in the transition from laminar to turbulent flow $[1,2]$. In Couette flow, with the inner cylinder only rotating at the rotation rate $\Omega$, the steady flow is laminar for low values of $\Omega$; for the critical value of the rotation rate $\Omega_{\mathrm{c}}$ and the corresponding Reynolds number $R_{\mathrm{c}}=\Omega_{\mathrm{c}} R_{1} d / v$ ( $v$ is the kinematic viscosity, $R_{1}, R_{2}$ are respectively the inner and outer cylinder radii, $d=R_{2}-R_{1}$ ), this flow becomes unstable and a new steady pattern of toroidal rings, the Taylor vortices, superimposed on the azimuthal laminar flow, is established. Then, the flow becomes time-dependent and the Taylor structure acquires azimuthal waves, which correspond to a single sharp frequency component $f_{1}$ in the velocity spectrum. For larger values of $\Omega\left(R / R_{\mathrm{c}}=\Omega / \Omega_{\mathrm{c}} \simeq 10\right)$, a second frequency component $f_{2}$

(*) Laboratoire associé au Centre National de la Recherche Scientifique. 
appears [3]. For $R / R_{\mathrm{c}} \simeq 12$, a broadband component centred at $f_{\mathrm{B}}=0.4 \Omega$ becomes apparent, this latter component is the only one which remains for $R / R_{\mathrm{c}} \gtrsim 20$ and appears as the signature of the turbulent flow [2]. For still larger values of $R$ and particular experimental conditions, Walden and Donnelly [4] have found a new sharp peak $f_{\mathrm{r}}$, which reemerges in the power spectrum. Barcilon et al. [5] and Koschmieder [6] have studied the turbulent flow for high values of $R$ ( $\simeq 200-300 R_{\mathrm{c}}$ ) and have observed through a visualization technique the appearance of a smaller scale structure, attributed to Görtler vortices by Barcilon et al.

The low value of the kinematic viscosity of normal helium $\left(v=\eta / \rho=2 \times 10^{-4} \mathrm{~cm}^{2} . \mathrm{s}^{-1}\right.$ i.e. two orders of magnitude lower than usual liquids) permits us to study easily its hydrodynamic behaviour at very high values of $R\left(\simeq 500 R_{\mathrm{c}}\right)$ with the experimentally imposed mechanical parameters of rotation $\left(d, R_{1}\right.$ and $\left.\Omega\right)$. Thus, the described results concern particularly the range of rotation which corresponds to the usually called «turbulent state». As soon as $d$ is sufficiently large $(d / R>0.08)$, the more striking result is the formation of a new ordered structure, characterized by a sharply defined periodic mode « $R$ » similar to the « wavy mode ».

1. Experimental procedure. - The experimental apparatus consists of two concentric cylinders : the radius of the stator is $R_{2}=2.650 \mathrm{~cm} \pm 0.001 \mathrm{~cm}$ and the radius of the rotor $R_{1}$ can be chosen between $2.55 \mathrm{~cm}$ to $2.15 \mathrm{~cm}$. For all values of $d$, the aspect ratio $\Gamma=l / d(l=$ cylinder height) can be taken between 2 and 12. In rotation experiments at liquid helium temperature, obtaining a stable rotation with a small dissipation is difficult; with our apparatus, the rotation rate $\Omega$ is constant to better than $\pm 0.3 \%$ in the range $10^{-1}-10 \mathrm{~Hz}$ with the gap $d$ constant to better than $\pm 3 \mu$ whatever the rotation $\Omega$. But the main difficulty is to assume a constant temperature of the helium liquid in the studied range $T_{0}=2.2 \mathrm{~K}-3 \mathrm{~K}(1 \mathrm{mK}$ corresponds to the threshold difference of temperature for Rayleigh-Bénard instabilities in helium liquid with a characteristic length of $2 \mathrm{~mm}$ ). To obtain this temperature stability, we control the main $\mathrm{He}$ bath to within $10^{-2} \mathrm{~K}$ by the regulation of the vapour pressure of liquid $\mathrm{He}$ with a mechanical manostat; a stainless steel chamber partially isolates the experimental cell from the main bath; the two cylinders are made in copper, and the stator is electronically regulated at a constant temperature $T_{1}=T_{0}+\varepsilon\left(\varepsilon \simeq 3 \times 10^{-2} \mathrm{~K}\right.$ to within $\left.10^{-6} \mathrm{~K}\right)$; the temperature of the liquid helium between the cylinders is thus maintained at the temperature $T_{1}$ with a stability better than $10^{-3} \mathrm{~K}$.

The detection system, somewhat different from the one used in preliminary results [7], is similar to the method used in hot wire anemometry : the detector is an Aquadag painted layer with a rectangular form ( $\simeq 1 \times 5 \mathrm{~mm}$ ), the larger dimension being parallel to the azimuthal flow, so that it is more sensitive to the departure from this main flow; it is heated by a d.c. power of typically $10^{-5} \mathrm{~W}$, such that its stationary temperature at $\Omega=0$ is greater than the liquid one $T_{1}$ by about $10^{-3} \mathrm{~K}$. When $\Omega$ differs from 0 , the heat of the probe is more and less scattered by the flow, therefore its temperature and resistance value $r$ depend on the local velocity field. We measure the detector resistance using a lock-in detector and an a.c. bridge; the bridge signal, proportional to

$$
\Delta r(t)=r(\Omega)-r(\Omega=0),
$$

is analysed using a HP 5420 A digital signal analyser. It is difficult to estimate the size of the probed volume, but we think that it expands on a radial dimension of about $1 \mathrm{~mm}$; thus by no means can the probe be considered as testing the boundary layer only.

This detection allows us to reach experimentally the threshold $\Omega_{\mathrm{c}}$ of the onset of the Taylor cells : these thresholds are determined by a small jump $\delta r(t)$ and the apparition of low frequency oscillations of the probe resistance; the determined values $\Omega_{\text {cexp }}=12 \mathrm{mHz}$ for $d=2 \mathrm{~mm}$ and $\Omega_{\text {cexp }}=35 \mathrm{mHz}$ for $d=1 \mathrm{~mm}$ correspond to a mean critical Taylor number

$$
T a_{\mathrm{cexp}}=2 \frac{\Omega_{\mathrm{c}}^{2}}{v^{2}} d^{3} R_{1} \simeq 4000
$$


this value is $20 \%$ higher than the theoretical one $\left(T a_{\mathrm{c}}=3370\right)$. All the reported results will be given as a function of the dimensionless measure of the rotation rate $T a / T a_{\mathrm{c}}$ or $R / R_{\mathrm{c}}$.

2. Experimental results. - 2.1 MODES « $B$ » AND « $R »$. - Let us now describe the evolution of the time dependence of the local velocity by the study of $r(t)$ and the corresponding power spectrum $P(f)$ as $R$ is increased, for $\Gamma=6$ and $d=2 \mathrm{~mm}$ (Fig. 1). For values of $R$ from $6 R_{\mathrm{c}}$ to $10 R_{\mathrm{c}}$ a mode is present, the frequency component of its velocity spectrum is broad and centred around $f_{\mathrm{B}} \simeq 0.4 \Omega$; its features (appearance, broadband, value) are similar to the mode" « $\mathrm{B}$ » seen in usual liquids after the sharp modes $f_{1}$ and $f_{2}$ [2], but it seems more important in our case ; this mode persists up to $R=39 R_{\mathrm{c}}$, although its behaviour changes with increasing $\Omega$ : gradually, high order harmonics of $f_{\mathrm{B}}$ appear and their amplitude is no longer decreasing but show a maximum around the seventh harmonic (Fig. $1 b$ ). In figure $1 c$, for $R / R_{\mathrm{c}}=37$, the fundamental mode

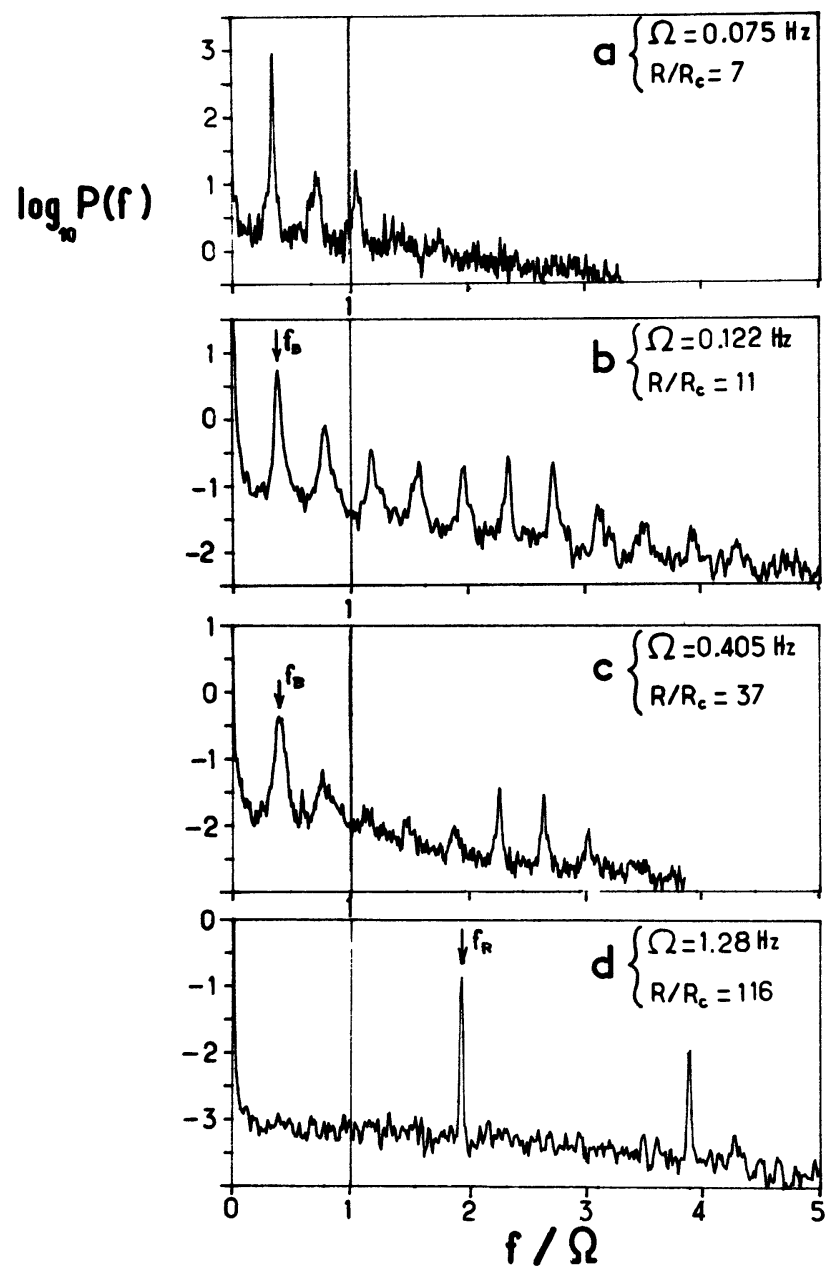

Fig. 1. - Frequency spectrum $P(f)$ of the detector time-dependent resistance $r(t)$ for increasing normalized Reynolds numbers $R / R_{\mathrm{c}}$. The abscisse scale is normalized to the rotation rate $\Omega$ for each spectrum and a logarithmic scale with arbitrary units is used for the vertical axis. Figures $1 a, 1 b, 1 c$ are related to the mode « $\mathrm{B}$ » for $R / R_{\mathrm{c}}=7,11,37$ (frequency $f_{\mathrm{B}}$ and its harmonics). In figure $1 d\left(R / R_{\mathrm{c}}=116\right)$, the sharp frequency components $f_{\mathrm{R}}$ and $f_{2 \mathrm{R}}$ characterize the mode « $\mathrm{R} »$; the modulation mode $f_{\mathrm{m}}$ is not easily seen in this figure. In all recordings, $d=2 \mathrm{~mm}, R_{2}=26.5 \mathrm{~mm}, \Gamma=6$ and $T \simeq 2.4 \mathrm{~K}$. 
$f_{\mathrm{B}}$ is always important, but only the 6th and 7th harmonics remain and are sharper [8]. For $R=R_{\mathrm{R}}=39 R_{\mathrm{c}}$, an abrupt transition occurs, the mode $" \mathrm{~B}$ » and its harmonics disappear, the velocity spectrum is only dominated by the sharp frequency component $f_{\mathrm{R}}$ and the harmonic $2 f_{\mathrm{R}}, 3 f_{\mathrm{R}}$ of a mode which we label « $\mathrm{R}$ »; this mode remains for values of $R / R_{\mathrm{c}}$ from 39 to the maximum rotation rate $\left(R / R_{\mathrm{c}} \simeq 500\right)$ and corresponds to the power spectrum recorded in figure $1 d\left(R / R_{\mathrm{c}}=116\right)$. Let us note at this point that the change in the background noise is not relevant [9].

Let us describe the main features of this mode « $R$ » :

- Its observation for $\Omega>\Omega_{\mathrm{R}}$ does not depend on previous history.

- As for the " wavy " modes $f_{1}$ [10], it can exist in many states so that its frequency $f_{\mathrm{R}}$ can be written $f_{\mathrm{R}}=n F_{\mathrm{R}}$ with generally $3<n<6(n=5,6$ are the most probable modes). All the experimental results are summarized in figure 2 , where we have plotted the $\Omega$-normalized frequency as a fonction of the $\Omega_{\mathrm{c}}$-normalized rotation rate for $\Gamma=6$ and $d=2 \mathrm{~mm}$; we see that for $R / R_{\mathrm{c}} \lesssim 150, n$ is not single valued and its value depends mainly how the rotation rate is established.

- For $R / R_{\mathrm{c}} \gtrsim 150$, the ratio $f_{\mathrm{R}} / n \Omega$ is nearly constant (in Fig. $2, f_{\mathrm{R}} / n \Omega=0.38$ ).

- Often, this mode is slightly modulated at a frequency $f_{\mathrm{m}}$ close in value to $f_{\mathbf{B}}$; this modulation corresponds in the velocity spectrum to two broad peaks at frequencies $f_{\mathrm{R}} \pm f_{\mathrm{m}}$. We have plotted in figure 2 the $\Omega$-dependence of this modulation mode $f_{\mathrm{m}}$; for large values of $R / R_{\mathrm{c}}$, this modulation is more effective and a frequency locking occurs between these two frequencies $f_{\mathrm{R}}$ and $f_{\mathrm{m}}$ (the ratio $f_{\mathrm{R}} / f_{\mathrm{m}}$ is often an integer as in Fig. 2).

Let us note at this point some similarities in this scenario with the one corresponding to $f_{1}$ and $f_{2}: f_{\mathrm{R}}$ plays the role of $f_{1}$ and $f_{\mathrm{m}}$ that of $f_{2}:$ we think that, at these high rotation rates appears a flow with two boundary layers localized near the cylinders and a central region with an almost constant mean velocity.

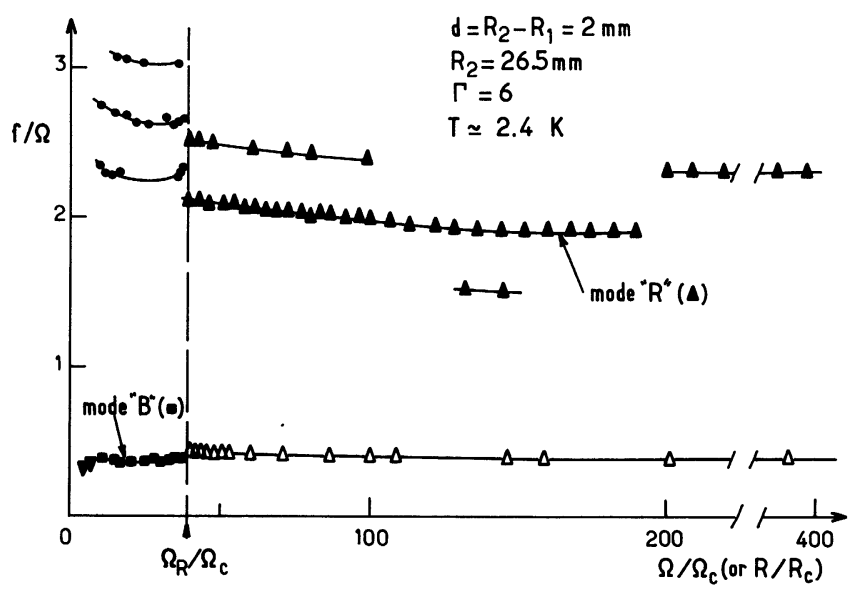

Fig. 2. - Frequency components observed in the spectra $P(f)$ obtained for $R / R_{\mathrm{c}}$ varying from 5 to 400 . We can see the $\Omega$-dependence of the frequencies $f_{\mathrm{B}}(\boldsymbol{\nabla})$ and $f_{\mathrm{R}}(\Delta)$. The points $(\boldsymbol{\bullet}, \Delta, \boldsymbol{\nabla})$ correspond respectively to the harmonics $6 f_{\mathrm{B}}, 7 f_{\mathrm{B}}, 8 f_{\mathrm{B}}$, to the modulation mode $f_{\mathrm{m}}$ and to the mode $f_{1}$.

2.2 VARIATION OF THESE MODES WITH $\Gamma, v$ AND $d$. - To have a better understanding of these results, we have studied the $\Gamma$-, $v$ - and $d$-dependence of this new evolution.

- For the gap $d=2 \mathrm{~mm}$, we have run various samples in which $\Gamma$ varies between 4 and 12 (corresponding to 4-12 rolls of theoretical dimension $d$ ). The qualitative evolution towards turbulence is the same as above, but the values of $f_{\mathrm{B}}, \Omega_{\mathrm{R}}, f_{\mathrm{R}} / n \Omega, f_{\mathrm{R}} / f_{\mathrm{m}}$ are somewhat modified. 
- We have studied the $v$-dependence of the experimental threshold $\Omega_{\mathrm{R}}$ for $d=2 \mathrm{~mm}$ and $\Gamma=6$; the results are plotted in figure $3 a$ and show a proportionality of $\Omega_{\mathrm{R}}$ with $v$ within a good approximation (as the threshold $\Omega_{\mathrm{c}}$ ).

- The dependence of $\Omega_{\mathrm{R}}$ on the gap $d$ is shown in figure $3 b: \Omega_{\mathrm{R}}$ increases as $d$ decreases and so sharply that for $d=1.5 \mathrm{~mm}$ and $d=1 \mathrm{~mm}$, we have not been able to see this transition up to maximum values of $\Omega / \Omega_{\mathrm{c}}$ (200 and 400 respectively); the velocity spectrum is then only characterized by $f_{\mathrm{B}}$ and its high order harmonics. We have not found any simple law for this dependence but this result seems to be related to the formation of boundary layers; if $d$ is large enough, the boundary layers can exist ; if not, the flow keeps a quasi-disordered structure.
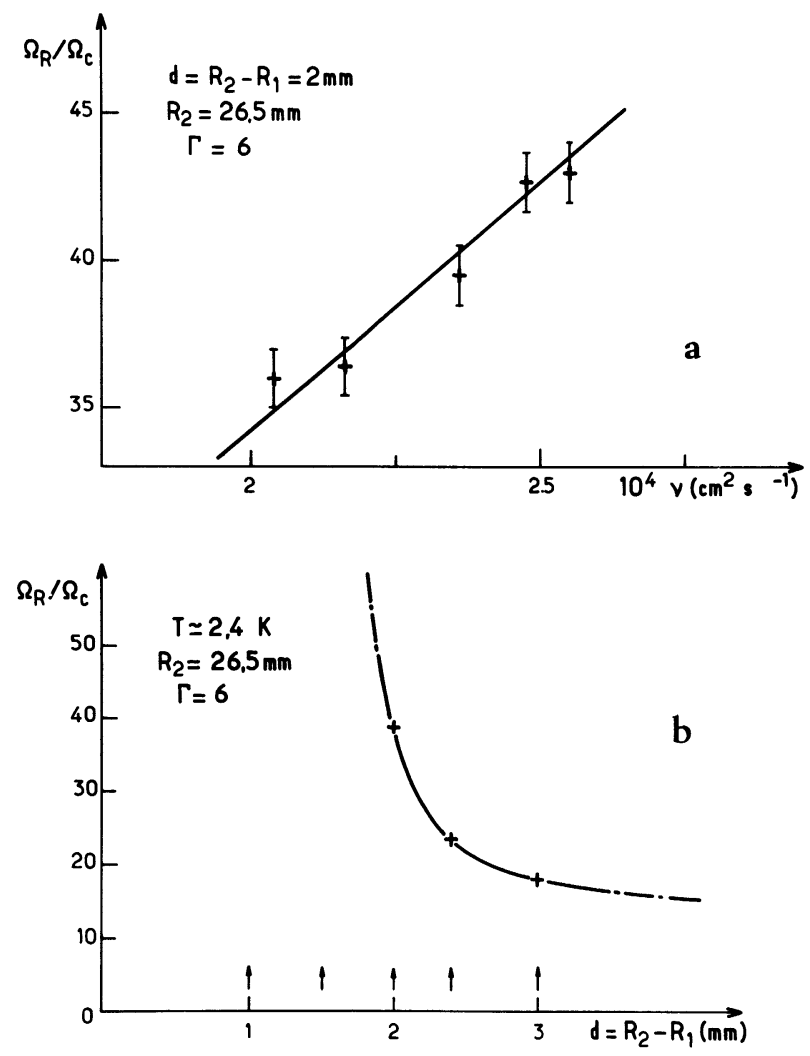

Fig. 3. - Normalized threshold $\Omega_{\mathrm{R}} / \Omega_{\mathrm{c}}$ of the onset of the mode « $\mathrm{R}$ » as a function of : a) The viscosity $v$ of liquid helium (the $v$ variation is obtained by increasing the temperature between $2.2 \mathrm{~K}$ and $2.6 \mathrm{~K}$ ). $b$ ) The gap $d=R_{2}-R_{1}$ (no threshold is observed for $d=1 \mathrm{~mm}$ and $d=1.5 \mathrm{~mm}$ ).

2.3 SEARCH FOR A THEORY. - The only result similar to ours is the reemergent wave observed in some cases in $\mathrm{CCl}_{4}$ [4] with $10<\Gamma<80$ and $28<R / R_{\mathrm{c}}<36$; the $\Omega$-dependence of its frequency $f_{\mathrm{r}}=1.435 \Omega$ agrees with our results for $n=4$. Moreover, beautiful experiments of visualization of the Couette flow $[5,6]$ give informations about our results.

Let us try now to describe a possible scenario for the appearance of $f_{\mathrm{B}}$ and $f_{\mathrm{R}}$. Barcilon et al. [5] conclude from their analysis that a smaller scale structure appears at sufficiently high Reynolds numbers and they attribute it to Görtler vortices. The dynamic of the structure could then manifests itself on two different length scales. On the length scale of the Taylor vortices, it would lead to the mode $f_{\mathrm{B}}$. On the smaller length scale of the Görtler vortices, it would lead to the presence of 
higher harmonics of $f_{\mathrm{B}}$ with large oscillating strength. Finally, the appearance of the mode « $\mathrm{R}$ » could be associated to a new structure of the convective pattern, somewhat different from the Taylor cells, with two well defined boundary layers where nearly all the variations of angular momentum take place [11] and a central flow with an angular momentum almost constant $\left(1 / 2 \Omega R_{1}^{2}\right)$. In this new structure, the central region at high $R / R_{\mathrm{c}}$ plays the role of the region between the cylinders at low $R / R_{\mathrm{c}}$; so the mode " $\mathrm{R}$ » would correspond to the "wavy mode " of the Taylor cells, its onset in Reynolds number $\left(R_{\mathrm{R}}=39 R_{\mathrm{c}}\right.$ for $\left.d=2 \mathrm{~mm}\right)$ being strongly displaced because the mean angular momentum gradient and the characteristic length are reduced. Evidently, this interpretation supposes that the boundary-layer thickness is smaller than $d$. From the logarithmic velocity profile and Taylor's experiments [12], Townsend [13] deduces that the region of nearly constant angular momentum is only possible if the gap is sufficiently large and more precisely if $R_{2} / R_{1}>1.08$; this condition corresponds in our case to $d>1.95 \mathrm{~mm}$, which agrees with the $d$-dependence of $\Omega_{\mathrm{R}}$. This agreement could be an indication of the boundary layers being turbulent but we cannot presently obtain experimental informations on this fact from the spectrum analysis, the background noise being essentially a thermal one [9].

Two main points remain :

- Why has this mode « $\mathrm{R}$ » not clearly been seen in other experiments $? R / R_{\mathrm{c}}$ not high enough ? $d / R_{1}$ too small ? This may be due to our small aspect ratio and in future experiments we intend to study the evolution of this mode when $\Gamma$ is being increased.

- In our experiments, the " wavy " mode $f_{1}$ was generally difficult to observe; when it was seen, its frequency was always $f_{1} \simeq \Omega / 3$ (one wavelength); when $R / R_{\mathrm{c}}$ increases from 3 to 10 , the periodic mode $f_{1}$ goes continuously into the mode « $\mathrm{B}$ » with a decreasing amplitude, a gradual broadening and a frequency evolving towards $0.4 \Omega$; the end of this evolution is clearly seen in figure $1 a$. When $f_{1}$ is not observed, the power spectrum always shows a large $f^{-n}$ noisy spectrum. The modulation of the waves, the mode $f_{2}$, was never seen. The stability of our very low rotation rates is perhaps responsible on these facts.

Acknowledgments. - We want to acknowledge A. Libchaber for stimulating discussions and C. Laroche for helpful assistance.

\section{References}

[1] COLES, D., J. Fluid Mech. 21 (1965) 385.

[2] Fenstermacher, P. R., Swinney, H. L. And Gollub, J. P., J. Fluid Mech. 94 (1979) 103.

[3] Gorman, M. and Swinney, H. L., Phys. Rev. Lett. 43(1979) 1871.

[4] Walden, R. W. and Donnelly, R. J., Phys. Rev. Lett. 42 (1979) 301.

[5] Barcilon, A., Brindley, J., Lessen, M. and MobBs, F. R., J. Fluid Mech. 94 (1979) 453.

[6] KosChMiEder, E. L., J. Fluid Mech. 93 (1979) 515.

[7] Wolf, P. E. and Perrin, B., Phys. Lett. 73A (1979) 324.

[8] Note added in proof : L. A. ReITH and H. L. SwinNEY have recently seen the qualitatively same effect at $R / R_{\mathrm{c}}=19.7$ (private communication).

[9] The essential background noise of the spectrum comes from the bolometer. Thus, it is difficult to get relevant informations from the change of this background noise as a function of Reynolds number. For example, the thermal contact of the bolometer could be improved at larger $R$, which would reduce the background noise (Fig. 1).

[10] This similarity between $f_{1}$ and $f_{\mathrm{R}}$ explains the interpretation of our preliminary results [7].

[11] Taylor, G. I., Proc. R. Soc. London A 151 (1935) 494.

[12] TAYlor, G. I., Proc. R. Soc. London A 157 (1936) 546.

[13] TownSEND, A. A., The structure of turbulent shear flow (Cambridge University Press) 1976. 